\title{
TLRs doing it for themselves
}
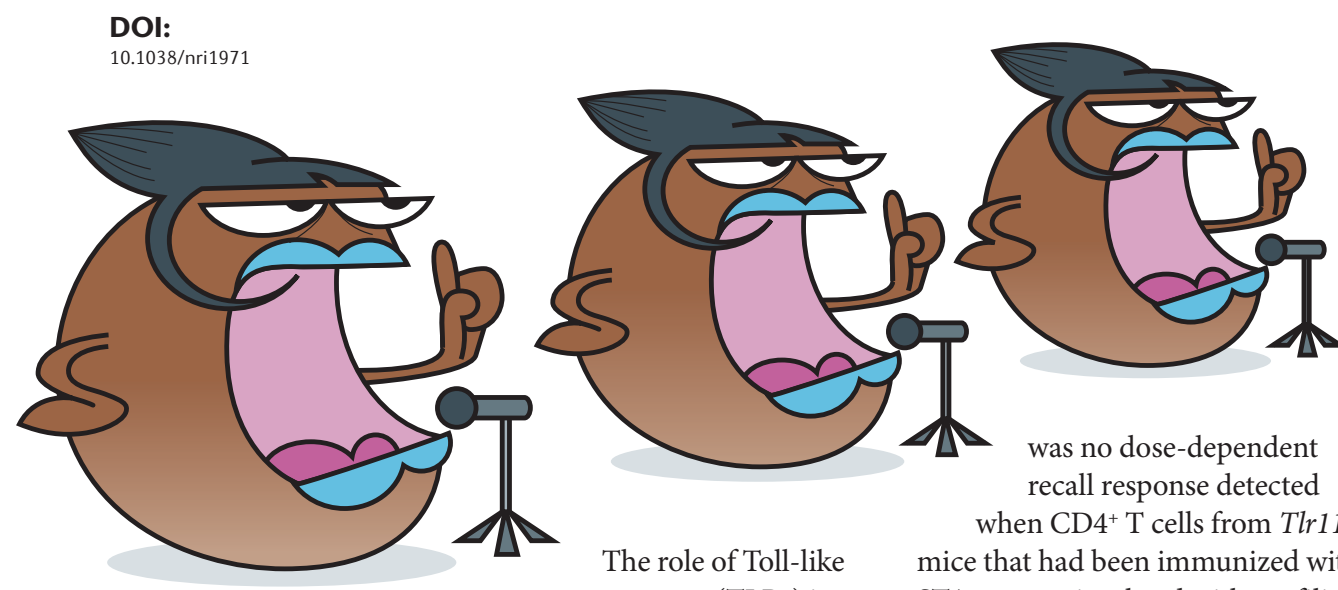

when $\mathrm{CD}^{+} \mathrm{T}$ cells from $\mathrm{Tlr} 11^{-1}$

The role of Toll-like receptors (TLRs) in

the activation of innate immune cells is well known, but their involvement in the presentation of antigen to $\mathrm{T}$ cells and the subsequent activation of those T cells is not well understood. Now, new research shows that TLR-mediated recognition of the parasite antigen profilin is required for its presentation to, and activation of, $\mathrm{CD}^{+} \mathrm{T}$ cells.

Profilin is a TLR11 ligand from the protozoan parasite Toxoplasma gondii, and this antigen selectively activates $\mathrm{CD} 8 \alpha^{+}$dendritic cells (DCs) in a MyD88 (myeloid differentiation primary-response gene 88)-dependent manner to produce interleukin-12. Because profilin is also a protein antigen that is presented to T cells, Yarovinsky et al. examined the ability of this antigen to activate $\mathrm{CD}^{+} \mathrm{T}$ cells following interaction with TLR11. Mice were immunized with a soluble extract of the tachyzoite stage of the parasite (STAg; which contains a complex mixture of microbial components and mimics parasite infection), then $\mathrm{CD} 4^{+} \mathrm{T}$ cells were stimulated with profilin in vitro. Profilin was found to be a dominant antigen in the $\mathrm{CD} 4^{+} \mathrm{T}$-cell response to T. gondii STAg. By contrast, there mice that had been immunized with STAg were stimulated with profilin. The CD4 ${ }^{+} \mathrm{T}$-cell response to profilin was also absent in $M y d 88^{-/-}$mice that had been immunized with STAg.

Therefore, the immunodominance of the antigen profilin depends on its recognition by TLR11 and signalling through MyD88.

To determine the cell population involved in the activation of profilinspecific $\mathrm{CD}^{+}{ }^{+} \mathrm{T}$ cells, differentiated profilin-specific $\mathrm{CD} 4^{+} \mathrm{T}$-cell clones were stimulated with various fractions of splenocytes from mice that had been immunized with STAg. The data showed that stimulation of these $\mathrm{CD} 4^{+} \mathrm{T}$ cells was restricted to CD $8 \alpha^{+}$DCs, and CD8 $\alpha^{+}$DCs from

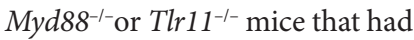
been immunized with STAg did not induce proliferation of these $\mathrm{T}$ cells.

The use of a mixed chimeric model, in which mice received bone-marrow cells from both MHC-class-II-deficient mice and

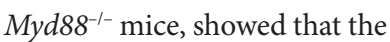
dual expression of both MyD88 and MHC class II molecules by the same bone-marrow-derived cell population is required for the activation of profilin-specific $\mathrm{CD} 4^{+} \mathrm{T}$ cells in vivo. The authors also generated chimeric mice that were reconstituted with a mixture of bone-marrow cells from wild-type mice and $M y d 88^{-/-}$mice, which express CD45.1 and CD45.2, respectively. These chimeric mice were then immunized with STAg, and 6 hours later, splenocytes were separated into $\mathrm{CD} 11 \mathrm{c}^{+}$populations of $M y d 88^{-/-}$and wild-type origin on the basis of expression of the CD45 allelic markers. Only the fraction that contained wild-type DCs could activate profilin-specific $\mathrm{CD} 4^{+} \mathrm{T}$ cells, indicating that the requirement for MyD88 is intrinsic to the DC itself. Therefore, the data indicate that the immunodominance of profilin depends on antigen recognition and signalling by the TLR and presentation of the antigen by the same DC.

Using fluorescently labelled profilin, the authors determined the role of TLR11 and MyD88 in the regulation of antigen uptake by DCs. The DC population from wild-type mice was found to contain fivefold more labelled cells than the population from either deficient mouse. So signals from the TLR can promote the uptake of protein antigens.

TLR-mediated recognition of the parasite antigen profilin therefore influences the presentation of profilin by $\mathrm{DCs}$ and regulates the activation of $\mathrm{CD}^{+} \mathrm{T}$ cells, establishing an additional mechanism by which TLR signalling can influence the adaptive immune response.

Olive Leavy

ORIGINAL RESEARCH PAPER Yarovinsky, F., Kanzler, H., Hieny, S., Coffman, R. L. \& Sher, A. Toll-like receptor recognition regulates immunodominance in an antimicrobial CD4 T cell response. Immunity 28 Sep 2006 (doi:10.1016/j.immuni.2006.07.015) 